\title{
THE EFFECT OF COMPRESSION AND EXPANSION OF PLASMA ON THE GENERATION OF SYNCHROTRON RADIATION
}

\author{
S. J. Gopasyuk, N. N. ERushev, and Y.I. NeshPor \\ (Crimean Astrophysical Observatory, Nauchny, Crimea, U.S.S.R.)
}

\begin{abstract}
We consider the variation of the synchrotron-flux density of relativistic electrons with the power law spectrum when the region of generation of this emission initially experiences a homogeneous compression and then an expansion. Ionization losses have been taken into account. The velocities of compression and expansion have been taken as constant. It is shown that in the cases of compression or expansion the flux density at a given frequency changes as

$$
S(t) \sim S_{0} K^{\gamma}(t)
$$

where $S_{0}=$ flux density before the compression, $\gamma=$ index of the power law spectrum, $K(t)=$ $(H(t)) /\left(H_{0}(t=0)\right)$, and $H$ is the magnetic-field strength. In the case of compression $K(t)>1.0$ and in the case of expansion $K(t)<1 \cdot 0$.

The results obtained are applied to an explanation of the increasing and decreasing parts of impulsive bursts of the centimeter range. Such a description of the impulsive bursts has allowed us to estimate both the parameters of the radiating region and the parameters of the differential energetic spectrum of relativistic electrons.
\end{abstract}

Kiepenheuer (ed.), Structure and Development of Solar Active Regions, 600. C. I.A.U. 